\section{Relationship between factor VIII activity, bleeds and individual characteristics in severe hemophilia A patients}

\author{
João A. Abrantes, ${ }^{1}$ Alexander Solms, ${ }^{2}$ Dirk Garmann, ${ }^{3}$ Elisabet I. Nielsen, ${ }^{1}$ \\ Siv Jönsson ${ }^{1}$ and Mats 0. Karlsson $^{1}$
}

${ }^{1}$ Department of Pharmaceutical Biosciences, Uppsala University, Uppsala, Sweden; ${ }^{2}$ Bayer, Berlin, Germany and ${ }^{3}$ Bayer, Wuppertal, Germany

\section{ABSTRACT}

$\mathrm{P}$ harmacokinetic-based prophylaxis of replacement factor VIII (FVIII) products has been encouraged in recent years, but the relationship between exposure (factor VIII activity) and response (bleeding frequency) remains unclear. The aim of this study was to characterize the relationship between FVIII dose, plasma FVIII activity, and bleeding patterns and individual characteristics in severe hemophilia A patients. Pooled pharmacokinetic and bleeding data during prophylactic treatment with BAY 818973 (octocog alfa) were obtained from the three LEOPOLD trials. The population pharmacokinetics of FVIII activity and longitudinal bleeding frequency, as well as bleeding severity, were described using non-linear mixed effects modeling in NONMEM. In total, 183 patients [median age 22 years (range, 1-61); weight $60 \mathrm{~kg}$ (11-124)] contributed with 1,535 plasma FVIII activity observations, 633 bleeds and 11 patient/study characteristics [median observation period 12 months (3.1-13.1)]. A parametric repeated time-tocategorical bleed model, guided by plasma FVIII activity from a 2-compartment population pharmacokinetic model, described the time to the occurrence of bleeds and their severity. Bleeding probability decreased with time of study, and a bleed was not found to affect the time of the next bleed. Several covariate effects were identified, including the bleeding history in the 12 -month pre-study period increasing the bleeding hazard. However, unexplained inter-patient variability in the phenotypic bleeding pattern remained large $(111 \% \mathrm{CV})$. Further studies to translate the model into a tool for dose individualization that considers the individual bleeding risk are required. Research was based on a post-hoc analysis of the LEOPOLD studies registered at clinicaltrials.gov identifiers: 01029340, 01233258 and 01311648.

\section{Introduction}

Hemophilia A is an X-linked hereditary bleeding disorder caused by the deficiency of coagulation factor VIII (FVIII). The severity of the disease is inversely correlated with the amount of factor that an individual is able to produce, and nearly half of the cases correspond to the severe laboratory phenotype (endogenous plasma FVIII activity levels $<1 \mathrm{IU} / \mathrm{dL}$ ). ${ }^{1,2}$ Prophylaxis with FVIII concentrates is currently considered the treatment of choice to decrease the frequency of bleeding and preserve musculoskeletal function. ${ }^{3.5}$ The population pharmacokinetics (PK) of FVIII products has been extensively studied, and it has been seen that the elimination half-life varies substantially between patients. ${ }^{6,7}$ Thus, the individual disposition of FVIII cannot be adequately predicted by average PK parameters and demographic characteristics, and PK-based dosing has been encouraged to optimize dosing regimens in the prophylactic setting. ${ }^{8-10}$ In brief, PK-based dosing relies on Bayesian estimation to estimate the individual PK parameters by combining patient information (dose, FVIII activity measurements and demographic characteristics, e.g. age, body weight) with information previously collected from a patient population by means of a population PK model. ${ }^{11,12}$ Based on the estimated
Haematologica 2020

Volume 105(5):1443-1453

\section{Correspondence:}

MATS 0. KARLSSON

mats.karlsson@farmbio.uu.se

Received: February 1, 2019.

Accepted: July 23, 2019.

Pre-published: August 1, 2019.

doi:10.3324/haematol.2019.217133

Check the online version for the most updated information on this article, online supplements, and information on authorship \& disclosures: www.haematologica.org/content/105/5/1443

(C)2020 Ferrata Storti Foundation

Material published in Haematologica is covered by copyright. All rights are reserved to the Ferrata Storti Foundation. Use of published material is allowed under the following terms and conditions:

https://creativecommons.org/licenses/by-nc/4.0/legalcode. Copies of published material are allowed for personal or internal use. Sharing published material for non-commercial purposes is subject to the following conditions:

https://creativecommons. org/licenses/by-nc/4.0/legalcode, sect. 3. Reproducing and sharing published material for commercial purposes is not allowed without permission in writing from the publisher. 
individual PK parameters, a patient-specific dosing regimen can be suggested. ${ }^{13}$ Recently, the shift from standard prophylaxis to PK-based prophylaxis was facilitated by the development of dosing tools, which are hemophiliaspecific for one (e.g. my PKFit, Shire Pharmaceutical Holdings Ireland Limited, Dublin, Ireland; wWw.mypkfit.com) or multiple (WAPPS-Hemo, McMaster University, Hamilton, Ontario, Canada, www.wappshemo.org) FVIII products, or generic tools (e.g. DoseMe LLC, Taringa Old, Australia; www.doseme-rx.com; InsightRX Inc., San Francisco, CA, USA, www.insightrx.com), which also support drugs from other clinical areas. $^{14}$

The choice of individual dosing regimens in PK-based prophylaxis is based on information about the individual $\mathrm{PK}$, but is also based on other components such as the patient's bleeding pattern, joint status or physical activity. From a PK perspective, the dose and dosing interval that generates an individual trough plasma FVIII activity above a certain target level, traditionally $1 \mathrm{IU} / \mathrm{dL}$, is selected. ${ }^{1,15}$ However, even though this level was not supposed to be an end in itself, ${ }^{16}$ but rather an orientation, until recently it was the main target used to build prophylaxis regimens. Several studies showed that some patients still bleed with higher trough values, while others do not bleed despite having trough values below $1 \mathrm{IU} / \mathrm{dL}$, suggesting that a "one-target-fits-all" strategy is not appropriate. ${ }^{16-22}$ Other measures of target FVIII exposure have been associated with bleeding, ${ }^{23}$ and different target levels for different patients that take into consideration individual lifestyle were recently suggested. ${ }^{24}$

One of the most important clinical endpoints to assess efficacy in hemophilia is bleeding frequency. This is often reported as the absolute number of bleeds during the study duration or annualized bleeding rate with the respective dispersion (e.g. range or standard deviation). ${ }^{25}$ Whereas these bleeding outcome measures may be useful to report efficacy, they are not adequate to study predictors of bleeding as they do not account for complex aspects, such as individual differences in FVIII disposition or bleeding phenotype, or time-varying factors, such as changes in dosing regimens or bleeding patterns. However, using an integrated model-based analysis overcomes these limitations. Repeated time-to-event modeling, an extension of parametric time-to-event survival analysis using non-linear mixed effects modeling, accounts for the occurrence of multiple events (e.g. bleeds) within an individual. ${ }^{26,27}$ This methodology enables the characterization of time-varying patterns in the occurrence of events (e.g. bleeding patterns changing over time) or predictors of events (e.g. FVIII activity) and has successfully been applied to several clinical areas, e.g. to describe the analgesic consumption in postoperative pain, ${ }^{28}$ or the time to the occurrence of epileptic seizures. ${ }^{29}$ This technique can be further extended to capture how consecutive events may be related to each other (Markovian dependence), or to include the severity of the events [repeated time-to-categorical event (RTTCE) modeling]. ${ }^{30}$

To better understand the link between prophylactic FVIII replacement therapy and bleeding patterns in patients with severe hemophilia A, the aim of this study was to characterize the relationship between FVIII doses, FVIII activity in plasma, and the occurrence of bleeds, as well as bleeding severity (mild, moderate or severe).

\section{Methods}

For a detailed description of the methods used, see the Online Supplementary Appendix.

\section{Patients and data}

This post-hoc analysis was based on dosing, PK, bleeding and patient characteristics data obtained from the three LEOPOLD trials (LEOPOLD I clinicaltrials.gov identifier: 01029340, LEOPOLD II clinicaltrials.gov identifier: 01233258 and LEOPOLD kids clinicaltrials.gov identifier: 01311648), ${ }^{31-33}$ evaluating efficacy, safety and PK of a full-length recombinant human FVIII product, BAY 81-8973 (octocog alfa, Kovaltry ${ }^{\circledR}$ ), ${ }^{34}$ in severe hemophilia A (endogenous FVIII activity $<1 \mathrm{IU} / \mathrm{dL}$ ) patients. Previously treated patients with no history of FVIII inhibitors, receiving on-demand or prophylactic treatment at screening, aged 12-64 years (LEOPOLD I and II) and $\leq 12$ years (LEOPOLD kids) were included. Single doses of 50 $\mathrm{IU} / \mathrm{kg}$ or 20-50 IU/kg 2-3 times/week (LEOPOLD I), 20-40 IU/kg 2-3 times/week (LEOPOLD II), and 25-50 IU/kg at least 2 times/week (LEOPOLD kids) were administered. The study protocols were reviewed and approved by each site's independent ethics committee or institutional review board.

Factor VIII activity was measured by the chromogenic assay. Bleeding episodes observed during prophylactic treatment were included in the analysis, which included spontaneous, traumarelated and untreated bleeds (i.e. bleeds not requiring FVIII infusions in addition to scheduled treatment), and unspecified events requiring FVIII treatment. Date and time of injection, and bleeding data (date, time, severity and location) were self-reported by the patient or caregiver using an electronic patient diary. A maximum of one bleed per calendar day was recorded, and spontaneous joint or muscle bleeds were not registered if occurring within 72 hours (h) of another bleed at the same site or respective infusion.

\section{Model development and assessment}

Model estimation was performed using non-linear mixed effects modeling in NONMEM ${ }^{\circledR} 7.4 .3 .{ }^{35}$ The PK and RTTCE models were estimated simultaneously and covariates integrated afterwards. Model assessment was based on scientific plausibility, changes in the objective function value (OFV, -2·log-likelihood), goodness-of-fit plots and precision of parameter estimates. For nested models, the likelihood ratio test was used [difference in $\mathrm{OFV}(\Delta \mathrm{OFV})>6.64$ considered significant at $=0.01,1$ d.f. $]$.

\section{Population pharmacokinetic model}

The population PK analysis started from the model by Garmann et al. using the corresponding set of data. ${ }^{36}$ The included association between lean body weight (LBW) and clearance (CL) and central volume of distribution (V1) was retained given the wide age range of patients. The previous model assumptions were comprehensively assessed.

\section{Repeated time-to-categorical bleed model}

The probability density of each bleed, as well as the probability associated with each severity was estimated from the observed time of bleeding and severity score (mild, moderate, severe) using a combination of parametric survival analysis and proportional odds model for ordered categorical data, ${ }^{26,27,37,38}$ i.e. the RTTCE model. ${ }^{30}$ The distribution of time of bleeds was explored using exponential, Weibull and Gompertz hazard functions. Inter-individual variability was considered on the overall bleeding hazard and on the logit transform of the severity probability. The censoring time for bleeds was set at the end of the individual bleeding observation period (right-censored observation).

The influence of individual plasma FVIII activity predicted from 
the estimated individual PK parameters and recorded dose information was explored on the baseline hazard using either a linear, exponential or maximum inhibition (Imax) model. Furthermore, a time-dependency between consecutive bleeds was assessed with a Markov hazard rate through a function depending on the time since the previous bleed.

The final RTTCE model, developed based on data of all bleeds, was re-estimated including only data concerning joint spontaneous bleeds from the studies LEOPOLD I and II to characterize the joint bleeding patterns in patients aged 12 years or older.

\section{Covariate analysis}

The correlation between covariates (patient and study characteristics), PK, bleeding hazard (all bleeds), and bleeding severity were evaluated using full random effects modeling. 39,40 This methodology allows the characterization of all model parametercovariate relationships in a single step and does not require imputation of pre-defined values when covariate data are missing. The covariates explored were: age, body weight, body mass index, lean body weight, race, von Willebrand factor (vWF), number of bleeds in the 12-month pre-study period, previous therapy history, on-demand or prophylaxis treatment, number of target joints for bleeds at study start obtained from the case report forms, ratio of the number of bleeds in the 12-month pre-study period to the number of target joints for bleeds at study start and during the study. In the original PK model development, ${ }^{36}$ vWF was not available; this covariate was, therefore, tested for the first time in this analysis. Relevant parameter-covariate relationships were identified by the correlation coefficient ( $r$ ), uncertainty of the effect size, and scientific plausibility.

\section{Results}

\section{Patients and data}

The final analysis included 1,535 FVIII activity observations from 183 patients, 633 bleeds from 172 patients, and 11 covariates. The median bleeding observation period was approximately 12 months for LEOPOLD I and II, and six months for LEOPOLD kids. Eleven patients had PK observations available but did not contribute with bleeding information because either they only received ondemand treatment $(n=5)$ or only participated in the PK part of the trial $(n=6)$. The median patient was a 22 -year old $60-\mathrm{kg}$ white male, with a vWF level of $104 \%$, one target joint at study start, and receiving prophylactic treatment before the study. In total, 116 patients ( $67 \%$ of total) had at least one bleed during the observation period (median 2, range 0-33), and the median time to first bleed was 48.2 days (range 14.5 hours-352 days). Descriptive statistics of study, patient characteristics and information on bleeding episodes are available in Table 1. (This information by age cohort is available in the Online Supplementary Appendix).

\section{Population pharmacokinetic model}

The PK component of the final model provided a good description of the PK data, similarly to the previously reported model. ${ }^{36}$ In addition to inter-individual variability on CL and V1, adding inter-individual variability on the residual error improved both model fit $(\Delta O F V=-199)$ and parameter precision. The median estimated individual CL was $1.80 \mathrm{dL} / \mathrm{h}$ (range $0.579-4.73 \mathrm{dL} / \mathrm{h}$ ) and $\mathrm{V} 1$ was $29.5 \mathrm{dL}$ (range 5.68-51.1 dL). The distribution of model-predicted plasma FVIII activity at the time of bleeding was strongly positively skewed with a median of $5.81 \mathrm{IU} / \mathrm{dL}$ (mean 11.6 $\mathrm{IU} / \mathrm{dL}$, range $<1.50-140 \mathrm{IU} / \mathrm{dL}$ ). The parameter estimates of the final model are available in Table 2.

\section{Repeated time-to-categorical bleed model}

A Gompertz hazard function with decreasing bleeding hazard over time provided an adequate description of the time-to-bleed data and was superior to the Weibull and exponential models. The effect of FVIII activity on the bleeding hazard was characterized by an Imax model $(P<0.001 ; \Delta O F V=-146)$, with full inhibition for high FVIII activity values. An exponential effect performed almost as well $(\triangle \mathrm{OFV}=-133)$, while a linear relationship performed substantially worse $(\Delta \mathrm{OFV}=-23)$. The final hazard equation was given by:

$$
\mathrm{h}(\mathrm{t})=\lambda \cdot e^{\mathrm{Y} \cdot(\mathrm{t}-1)} \cdot\left(1-\frac{\mathrm{FVIII}(\mathrm{t})}{\mathrm{FVIII}(\mathrm{t})+\mathrm{IF} 50}\right) \cdot e^{\eta}
$$

where $h(t)$ is the time-varying bleeding hazard, $\lambda$ and $\gamma$ are the scale and shape factors of the Gompertz distribution, FVIII( $t$ ) is the individual PK model-predicted FVIII activity at time $t$, IF50 is the FVIII activity resulting in halfmaximum inhibition of the hazard, and $\eta$ is a log-normally distributed random effect describing the unexplained inter-individual variability of the bleeding hazard in the population. Instead of representing the bleeding hazard when FVIII activity in plasma is zero, $\lambda$ and IF50 were reparametrized to represent the bleeding hazard when plasma FVIII activity was $0.5 \mathrm{IU} / \mathrm{dL}$ and $20 \mathrm{IU} / \mathrm{dL}\left(\lambda_{0.5 \mathrm{IU} / \mathrm{dL}}\right.$ and $\lambda_{\text {zoIU/dL, }}$ respectively) one year after study start. The assessment of a time dependency between consecutive bleeds, given by a transient effect where the occurrence of a bleed changed the bleeding hazard of a new bleed, could not be identified ( $P>0.05$ for Markov component). The estimated probability of a bleed during the study to be mild, moderate or severe was 39.6, 55.7 and $4.72 \%$, respectively.

The observed Kaplan-Meier curves for the first three bleeding episodes and the $95 \%$ confidence interval (CI) of the model predictions, showing how well the model described the data, are presented in Figure 1, and the parameter estimates of the final model are available in Table 2. An additional model diagnostic plot is available in the Online Supplementary Appendix (Online Supplementary Figure S1).

The re-estimated model accounting for the spontaneous joint bleeding information only was found to describe the data well (Online Supplementary Figure S2), and the final parameter estimates are available in Table 3. As expected, when including only spontaneous joint bleeds, the bleeding hazard as well as the IF50 parameter decreased, reflecting less frequent events and higher potency for the replacement therapy, respectively.

\section{Covariate analysis}

The estimated correlations between the model parameters (CL, V1, PK residual error, bleeding hazard and bleeding severity including all bleeds) and the co-variates are illustrated in Figure 2. The strongest relationships found were: vWF on CL ( $r=-0.54$; decreased unexplained interindividual variability by $4.7 \%$ ) and number of bleeds in the 12 -month pre-study period on the bleeding hazard $(\mathrm{r}=0.45$; decreased unexplained inter-individual variability by $15 \%$ ). The effect of LBW on CL and V1 was included $a$ 
Table 1. Patients' characteristics, treatment and bleeding data by study for patients with plasma factor VIII (FVIII) observations enrolled in the bleeding observation period.

\begin{tabular}{|c|c|c|c|c|}
\hline Study & LEOPOLD I & LEOPOLD II & LEOPOLD kids & Total \\
\hline Patients with PK and bleeding data, $\mathrm{n}$ (\% of total) & $62(36)$ & $59(34)$ & $51(30)$ & $172(100)$ \\
\hline \multicolumn{5}{|l|}{ Duration of bleeding observation period, months } \\
\hline Mean \pm SD & $11.8 \pm 1.53$ & $12.2 \pm 0.165$ & $6.12 \pm 0.544$ & $10.3 \pm 2.86$ \\
\hline Median [range] & $12.0[3.08-13.1]$ & $12.2[11.7-12.5]$ & $6.09[3.78-7.22]$ & $12.0[3.08-13.1]$ \\
\hline \multicolumn{5}{|l|}{ Individual FVIII dose during treatment period (IU/kg) } \\
\hline Mean \pm SD & $38.4 \pm 9.24$ & $38.9 \pm 9.37$ & $36.2 \pm 10.5$ & $38.2 \pm 9.60$ \\
\hline Median [range] & $37.9[4.25-80.9]$ & $38.7[5.85-199]$ & $34.1[19.2-106]$ & 37.9 [4.30-199] \\
\hline \multicolumn{5}{|l|}{ Patients' characteristics } \\
\hline \multicolumn{5}{|l|}{ Age, years } \\
\hline Mean \pm SD & $31.5 \pm 12.7$ & $29.0 \pm 11.1$ & $6.59 \pm 2.94$ & $23.2 \pm 14.9$ \\
\hline Median [range] & $30.0[12.0-61.0]$ & $27.0[14.0-59.0]$ & $6.00[1.00-11.0]$ & $22.0[1.00-61.0]$ \\
\hline \multicolumn{5}{|l|}{ Weight, kg } \\
\hline Mean \pm SD & $76.7 \pm 17.3$ & $64.9 \pm 13.2$ & $25.9 \pm 10.8$ & $57.6 \pm 25.5$ \\
\hline Median [range] & $76.0[39.0-124]$ & $64.0[46.0-98.0]$ & $22.6[11.0-59.0]$ & $60.0[11.0-124]$ \\
\hline \multicolumn{5}{|l|}{ Lean body weight, ${ }^{a} \mathrm{~kg}$} \\
\hline Mean \pm SD & $58.4 \pm 8.88$ & $52.8 \pm 7.18$ & $23.3 \pm 8.66$ & $46.0 \pm 17.1$ \\
\hline Median [range] & $58.5[35.5-79.2]$ & $52.2[40.9-68.4]$ & $20.8[9.25-48.9]$ & $50.9[9.25-79.2]$ \\
\hline \multicolumn{5}{|l|}{ Body mass index, ${ }^{\mathrm{a} g} \cdot \mathrm{m}^{-2}$} \\
\hline Mean \pm SD & $25.2 \pm 4.63$ & $21.4 \pm 3.94$ & $16.4 \pm 2.55$ & $21.3 \pm 5.25$ \\
\hline Median [range] & $25.2[16.2-38.3]$ & $20.8[15.0-30.9]$ & $15.7[13.0-24.6]$ & $20.3[13.0-38.3]$ \\
\hline \multicolumn{5}{|l|}{ von Willebrand factor level, $\%$} \\
\hline Mean \pm SD & $107 \pm 34.3$ & $110 \pm 38.9$ & $\mathrm{NA}$ & $109 \pm 36.5$ \\
\hline Median [range] & $103[53.0-200]$ & 104 [43.0-242] & $\mathrm{NA}$ & $104[43.0-242]$ \\
\hline \multicolumn{5}{|l|}{ Race, n (\%) } \\
\hline White & $55(89)$ & $30(51)$ & $47(92)$ & $132(77)$ \\
\hline Black & $4(6.5)$ & $1(1.7)$ & $3(5.9)$ & $8(4.7)$ \\
\hline Asian & $0(0.0)$ & $23(39)$ & $0(0.0)$ & $23(13)$ \\
\hline Hispanic & $2(3.2)$ & $5(8.5)$ & $1(2.0)$ & $8(4.7)$ \\
\hline Unknown & $1(1.6)$ & $0(0.0)$ & $0(0.0)$ & $1(0.60)$ \\
\hline \multicolumn{5}{|l|}{ Treatment history, n (\%) } \\
\hline On-demand & $11(18)$ & $59(100)$ & $11(22)$ & $81(47)$ \\
\hline Prophylaxis & $51(82)$ & $0(0.0)$ & $40(78)$ & $91(53)$ \\
\hline \multicolumn{5}{|l|}{ N. of target joints at study start } \\
\hline Median [range] & $1[1-4]$ & $2[1-5]$ & $1[1-2]$ & $1[1-5]$ \\
\hline \multicolumn{5}{|l|}{ Summary of bleeding episodes } \\
\hline Total number of bleeds, n (\% of total) & $240(38)$ & $292(46)$ & $101(16)$ & $633(100)$ \\
\hline Patients with at least one bleed, $n$ (\% of total) & $45(39)$ & $43(37)$ & $28(24)$ & $116(100)$ \\
\hline \multicolumn{5}{|l|}{ Individual n. of bleeds } \\
\hline Median [range] & $2[0-26]$ & $2[0-33]$ & $1[0-9]$ & $2[0-33]$ \\
\hline \multicolumn{5}{|l|}{ Time to first bleed, days } \\
\hline Mean \pm SD & $91.8 \pm 96.7$ & $63.9 \pm 66.0$ & $58.0 \pm 46.6$ & $73.3 \pm 76.8$ \\
\hline Median [range] & $49.1[1.00-351.9]$ & $43.0[0.606-265]$ & $5.00[1.17-182]$ & $48.2[0.606-352]$ \\
\hline \multicolumn{5}{|l|}{ Bleed type, n (\%) } \\
\hline Spontaneous & $155(65)$ & $209(72)$ & $24(24)$ & $388(61)$ \\
\hline Non-spontaneous & $85(35)$ & $83(28)$ & $77(76)$ & $245(39)$ \\
\hline \multicolumn{5}{|l|}{ Spontaneous bleed location, n (\%) } \\
\hline Joint & $131(55)$ & $189(65)$ & $8(7.9)$ & $328(52)$ \\
\hline No joint & $109(45)$ & $103(35)$ & $93(92)$ & $305(48)$ \\
\hline \multicolumn{5}{|l|}{ Bleed severity, n (\%) } \\
\hline Mild & $123(51)$ & $120(41)$ & $52(52)$ & $295(47)$ \\
\hline Moderate & $91(38)$ & $139(48)$ & $46(46)$ & $276(44)$ \\
\hline Severe & $26(11)$ & $33(11)$ & $3(3.0)$ & $62(9.8)$ \\
\hline \multicolumn{5}{|l|}{ N. of bleeds in the 12 months prior to study start ${ }^{\mathrm{e}}$} \\
\hline Median [range] & $5.5[0-55]$ & $36[3-106]$ & $4[0-55]$ & $11[0-106]$ \\
\hline
\end{tabular}


A

Strata + Bleed $1+$ Bleed $2+$ Bleed 3

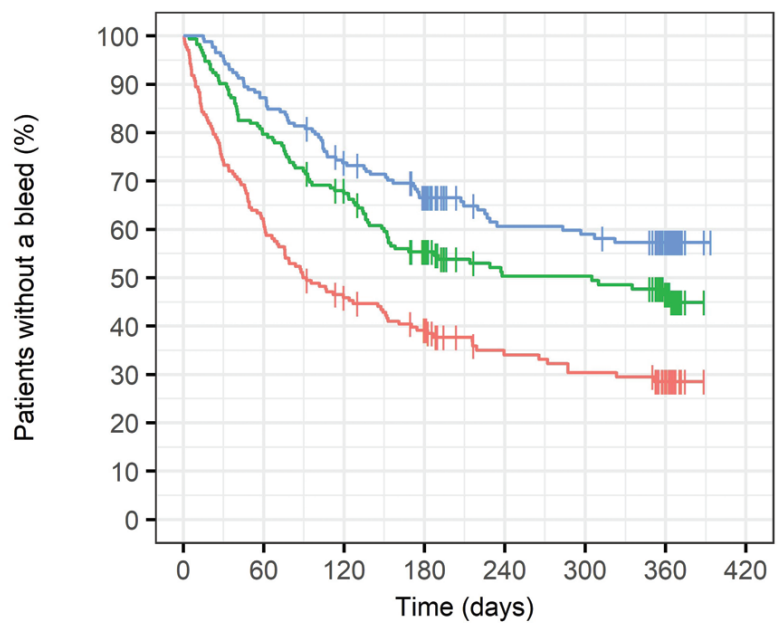

Cumulative number of bleeds

\begin{tabular}{|c|c|c|c|c|c|c|c|c|}
\hline \multirow[t]{4}{*}{ (0 Bleed 1 - } & 0 & 66 & 93 & 104 & 110 & 114 & 116 & 116 \\
\hline & 0 & 35 & 56 & 76 & 82 & 82 & 86 & 87 \\
\hline & 0 & 22 & 45 & 57 & 64 & 66 & 68 & 68 \\
\hline & 0 & 60 & 120 & 180 & 240 & 300 & 360 & 420 \\
\hline
\end{tabular}

B

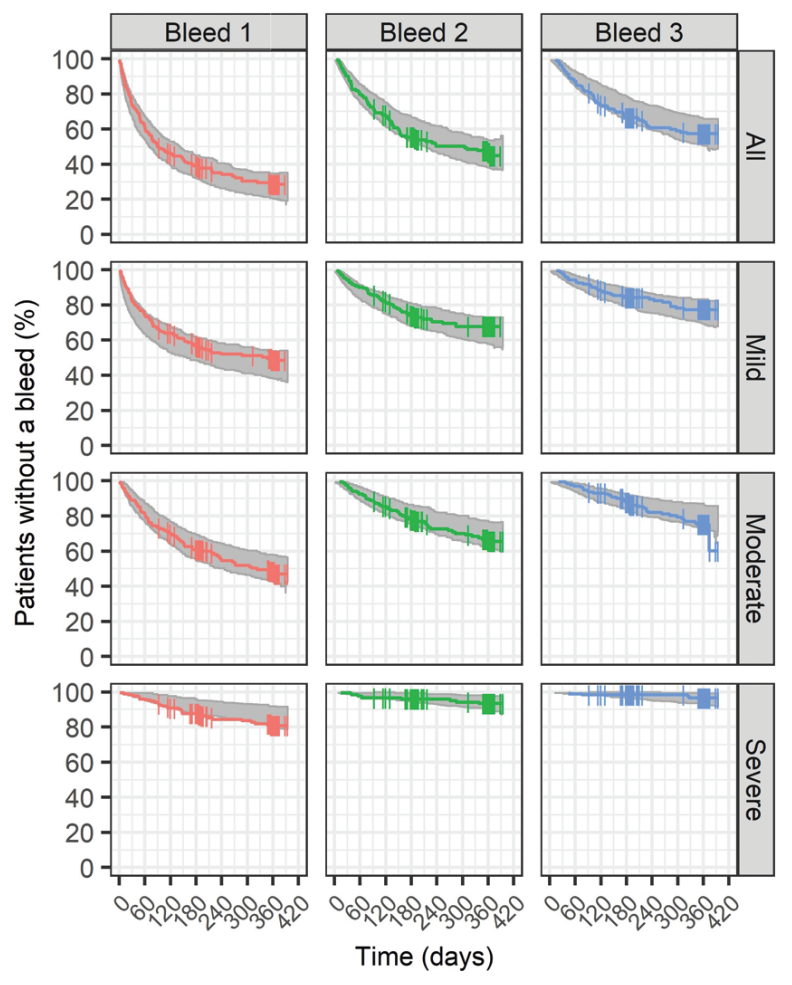

Figure 1. Observed and model-predicted Kaplan-Meier curves depicting the percentage of bleed-free patients versus time after start of the LEOPOLD studies, for the first, second and third individual bleed. (A) Observed Kaplan-Meier curves (plot) and cumulative number of bleeds throughout time (table). (B) Observed KaplanMeier curves by number of bleeds (first, second or third in the study) and bleeding severity (mild, moderate or severe) overlaid with the $95 \%$ confidence interval of the model-predicted Kaplan-Meier curves (shaded area), based on 200 simulations. Vertical lines indicate that a patient was censored.

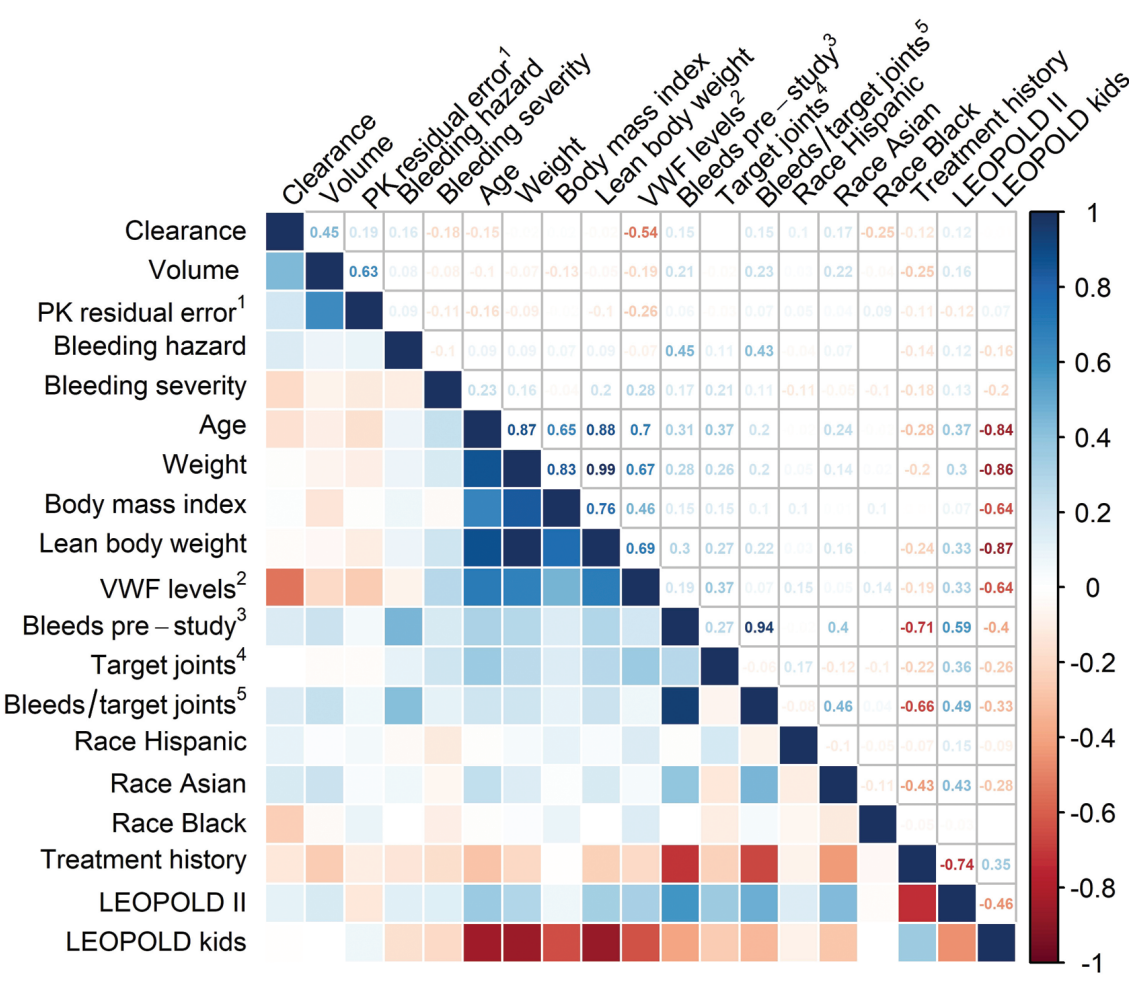

Figure 2. Correlation matrix illustrating the correlations between model parameters and observed study or patient characteristics (covariates). Each square illustrates the correlation between two variables (model parameters or covariates). The same variables are represented in the rows and columns, and the diagonal line shows that each variable correlates perfectly with itself. The first five rows/columns depict the relationship between the model parameters associated with inter-individual variability [clearance, central volume of distribution, pharmacokinetic (PK) residual error, bleeding hazard and bleeding severity] and the covariates, and the remaining rows/columns represent the correlations between the observed covariate values (on the log-scale). The matrix is symmetrical, and the correlations below the main diagonal line are represented with colors. The darker the color, the stronger the interdependence of the two variables, with strong negative correlations represented in dark red $(r=-1)$, i.e. one variable increases as the other decreases, and strong positive correlations represented in dark blue $(r=+1)$, i.e. one variable increases as the other increases. Correlations above the main diagonal line are represented with numbers detailing the correlation coefficient values ( $r$ ). ${ }^{1}$ Unexplained inter-individual variability on the residual error. '2VWF: von Willebrand factor. ${ }^{3}$ Number of bleeds in the 12-month pre-study period. ${ }^{4}$ Number of target joints for bleedings at study start. ${ }^{5}$ Ratio of the number of bleeds in the 12 months prestudy period to the number of target joints for bleedings at study start. 
priori in the PK structural model and the absence of a correlation between LBW on CL or V1 in the covariate analysis indicates that the relationship was well captured by the model. Other characteristics showed lower correlation coefficients, namely, Black race and Asian race decreasing and increasing $\mathrm{CL}(\mathrm{r}=-0.25$ and 0.17$)$, respectively, and age and $\mathrm{vWF}$ being positively correlated to the severity of bleeding $(r=0.23$ and 0.28$)$. In addition, correlations were identified between model parameters (e.g. CL and V1, $r=0.45$ ) and between covariates (e.g. treatment history and number of bleeds pre-study period, $\mathrm{r}=-0.71$ ).

The effect sizes of the most relevant parameter-covariate interactions are shown in Figure 3. The number of bleeds in the 12-month pre-study period correlating with the bleeding hazard was the most relevant interaction found. Compared to a mean patient with 8.2 bleeds in the pre-study period, a patient who had one bleed ( $5^{\text {th }}$ percentile of the observed data) or 84 bleeds $\left(95^{\text {th }}\right.$ percentile) pre-study was found to have a 54\% lower (95\% CI: 40-65) or $147 \%$ higher ( $95 \% \mathrm{CI}: 79-226)$ hazard, respectively. These values translate into a bleeding hazard $\left(\lambda_{0.5 \text { IU/dL }}\right)$ of 1.30 year $^{-1}$ (95\%CI: 0.99-1.68) (1 pre-study bleed), and 6.97 year $^{-1}$ (95\% CI: 5.05-9.18) (84 pre-study bleeds). von Willebrand factor levels and race were found to correlate with CL. A patient with a vWF level of $64 \%\left(5^{\text {th }}\right.$ percentile $)$ or $179 \%\left(95^{\text {th }}\right.$ percentile) had an estimated CL of $2.30 \mathrm{dL} / \mathrm{h}$ (95\% CI: 2.17-2.45; terminal half-life $11.6 \mathrm{~h}$ ) and $1.59 \mathrm{dL} / \mathrm{h}$ (95\% CI: 1.47-1.69; terminal half-life 16.5 h), respectively. Patients of Black race had an estimated CL of $1.41 \mathrm{dL} / \mathrm{h}$ (95\% CI: 1.16-1.71), Asian $2.21 \mathrm{dL} / \mathrm{h}$ (95\% CI: 1.97-2.48), and Caucasian $1.93 \mathrm{dL} / \mathrm{h}$ (95\%CI:1.80-2.07). A modest correlation was identified between vWF and bleeding severity, with a patient with a vWF level of $64 \%$ having a probability of having a moderate or severe bleed of $57 \%$ (95\% CI: 55-59), while the corresponding probability for a patient with a vWF level of $179 \%$ was $68 \%$ (95\% CI: 62 75); a similar trend was found for age.

\section{Simultaneous model predictions}

An illustration of the observed plasma FVIII activity and time-to-bleed data for three patients, and the respective model-based predictions are available in Figure 4.

\section{Variability in the bleeding hazard}

The inclusion of all available covariates in the model resulted in a decrease of $25 \%$ in the inter-individual variability on the bleeding hazard, with a final unexplained inter-individual variability value of $111 \%$. Figure 5 shows how this variability translates into bleeding frequency by using the model to simulate the occurrence of bleeds during one year in a group of virtual patients with median characteristics, following doses leading to a given plasma FVIII activity trough value: 1, 3, 5 and 15 IU/dL. Plasma FVIII activity-time profiles with higher trough values were linked to lower bleeding hazards, leading to an expected lower simulated bleeding frequency. The median cumulative number of simulated bleeds for the given dosing scenarios ranged between 0.745 (FVIII activity trough 15 IU/dL) and 2.73 (FVIII activity trough $1 \mathrm{IU} / \mathrm{dL}$ ) bleeds, and the $90^{\text {th }}$ percentile, representing the inter-patient variability on the bleeding hazard (underlying bleeding tendency), ranged between 3.00 and 11.2 bleeds.

\section{Discussion}

We developed a comprehensive mathematical model describing the relation between the dose of a recombinant FVIII product, plasma FVIII activity, the bleeding outcome as well as severity and the correlation with covariates, fol-

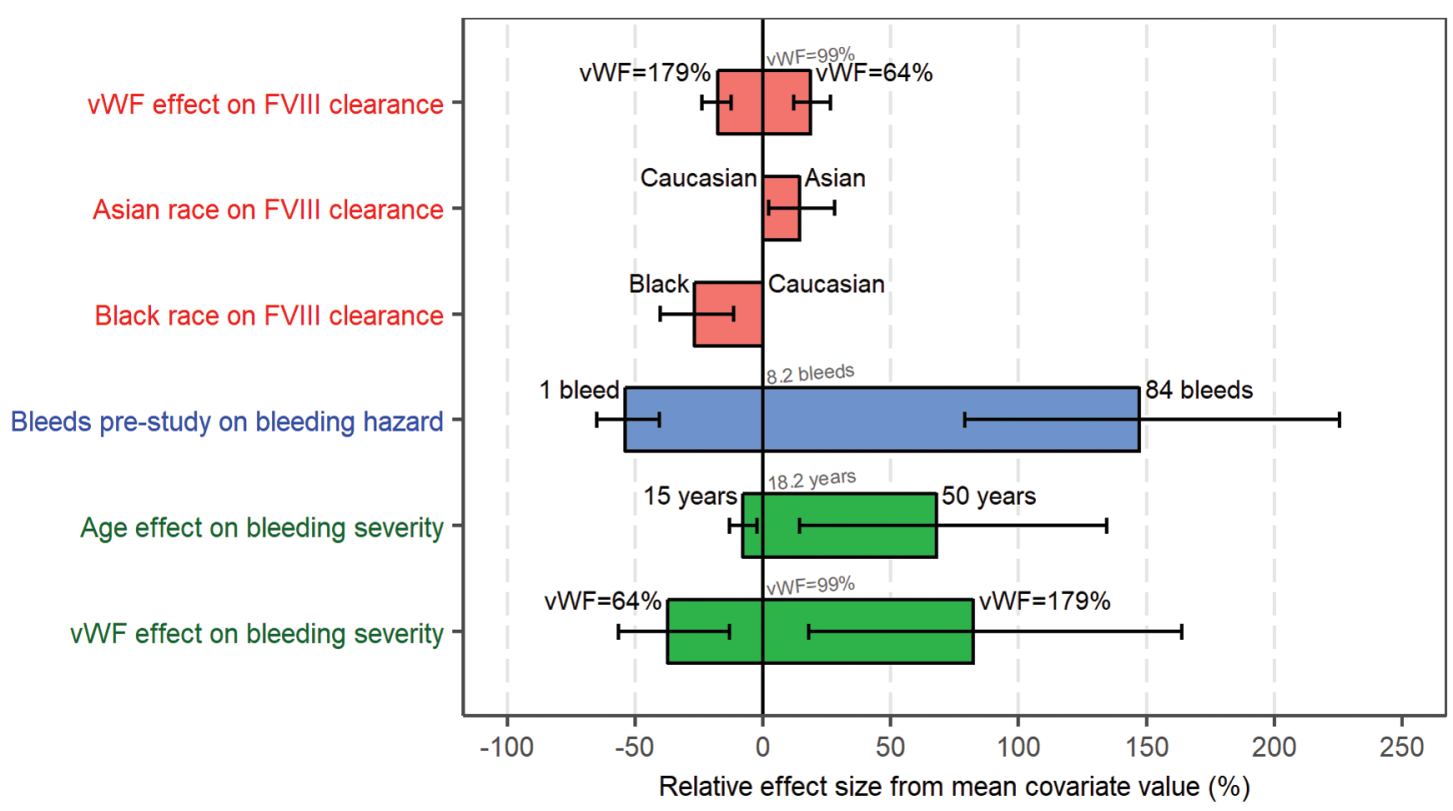

Figure 3. Effect sizes of the most relevant covariates on the estimated model parameters when compared to the mean covariate value, with uncertainty. For continuous covariates (vWF: von Willebrand factor, bleeds pre-study period, and age) the effects at the $5^{\text {th }}$ and $95^{\text {th }}$ percentiles of the covariates are shown, and the geometric mean value is represented in gray. For the categorical covariate race, the effect of the presented category is compared to the most frequent category. The black error bars represent the $95 \%$ confidence interval given by the uncertainty of the estimated model parameters. FVIII: factor VIII. 
lowing prophylactic treatment in patients with severe hemophilia A. To our knowledge, this is the first reported model characterization including longitudinal bleeding events for a replacement FVIII product. The model was based on observed data of plasma FVIII activity (PK component), bleeding time and severity (RTTCE component) and covariates, collected during the LEOPOLD studies. The final model satisfactorily described plasma FVIII activity and time- and PK-related probability of bleeding.
Besides LBW (included a priori influencing CL and V1), vWF and race correlated with CL, number of bleeds in the 12 -month pre-study period correlated with the bleeding hazard, and age and vWF affected the severity of bleeding. However, even after identifying these covariate effects, the unexplained inter-individual variability on the bleeding hazard was high.

The covariate analysis allowed the exhaustive assessment of the clinical relevance of the correlation between

- PK observation $\times$ Bleed observation — Individual model predictions - - Cumulative observation
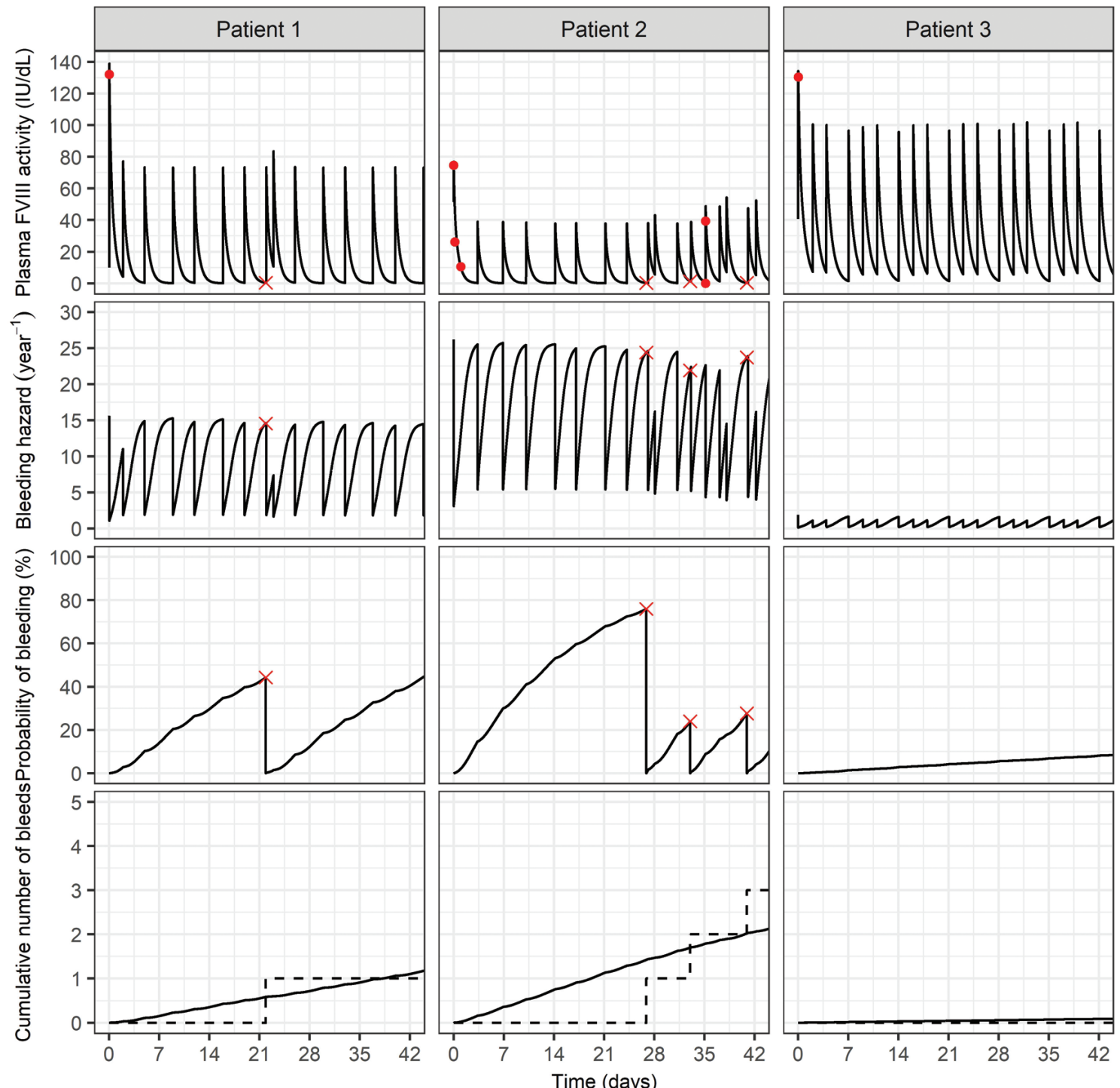

Figure 4. Illustration of the observed pharmacokinetics (PK), observed time-to-bleed data and model predictions (including co-variates) for three illustrative patients during the first six weeks in the study. Patient 1 participated in the LEOPOLD I trial and was 44 years old, weighed $107 \mathrm{~kg}$, received prophylactic treatment before the study started and had six bleeds in the 12 months pre-study period; a PK sample was collected 25 min post-dose on day 1 ; a bleed occurred on day 22. Patient 2 participated in the LEOPOLD kids trial and was 11 years old, weighed $40 \mathrm{~kg}$, received prophylactic treatment before the study started and had 15 bleeds in the 12 months pre-study period; PK samples were collected at 0.6, 4.25 and $24.2 \mathrm{~h}$ post-dose on day 1 , and pre-dose and $0.5 \mathrm{~h}$ post-dose on day 35 . Bleeds occurred on days 27, 33 and 41. Patient 3 participated in the LEOPOLD II trial and was 19 years old, weighed $68 \mathrm{~kg}$, received on-demand treatment before the study started and had 36 bleeds in the 12 months pre-study period; a PK sample was collected at 25 min post-dose on day 1; no bleeds were observed. The first row shows the PK observations (circles), respective model predictions (solid lines) and the time of bleeds (crosses). At the time of the bleeds, the model-predicted factor VIII (FVIII) activity values were $0.38 \mathrm{IU} / \mathrm{dL}$ (patient 1) and $0.30,1.39$ and $0.367 \mathrm{IU} / \mathrm{dL}$ (patient 2), all values below the lower limit of quantification. The second row depicts the predicted individual bleeding hazard, which is inversely correlated with plasma factor VIII activity, and decreases throughout the time of the study. The third row depicts the individual probability of bleeding, which was calculated based on the individual bleeding hazard (the higher the hazard, the faster the increase in the probability of bleeding) and was reset to zero every time a bleed occurred. The fourth row shows the observed and predicted cumulative number of bleeds over time. Further details on the mathematical derivation of the curves related to bleeding can be found in the Online Supplementary Appendix. 
Table 2. Parameter estimates for the simultaneous estimation of the pharmacokinetic and repeated time-to-categorical event (RTTCE) subcomponents of the final model.

\begin{tabular}{lcc} 
Parameters & Estimate & RSE (\%) \\
PK model & & \\
Structural model & & \\
CL, dL/h & 1.93 & 3.54 \\
V1, dL & 30.3 & 1.72 \\
Q, dL/h & 1.69 & 23.0 \\
V2, dL & 6.29 & 8.79 \\
\hline
\end{tabular}

\section{Covariate parameters}

Effect of LBW on $\mathrm{CL}^{\mathrm{a}}$

Effect of LBW on V1 ${ }^{\mathrm{a}}$

0.646

0.958

10.0

Inter-individual variability

\begin{tabular}{lll} 
CL, \%CV & $30.2^{\mathrm{b}}$ & $7.31^{\mathrm{c}}$ \\
$\mathrm{Vl}, \% \mathrm{CV}$ & $15.1^{\mathrm{b}}$ & $8.75^{\mathrm{c}}$ \\
Residual error, \%CV & $63.1^{\mathrm{b}}$ & $7.23^{\mathrm{c}}$ \\
\hline
\end{tabular}

Residual variability

Additive, IU/dL

1.52

11.0

Proportional, \%CV

20.3

5.52

RTTCE model (all bleeds)

Structural model

$\lambda_{0.5 \mathrm{IU} / \mathrm{dL}}$, year $^{-1}$

2.82

17.6

$\lambda_{20 \text { IU/dL, }}$ year $^{-1}$

1.00

$\lambda_{\text {(0 IU/dL, 1 year) }}(\text { derived) })^{\mathrm{d}}$, year ${ }^{-1}$

2.96

IF50 (derived) ${ }^{\mathrm{d}}$, IU/dL

$\gamma$, year $^{-1}$

10.2

$b_{1}$

$-0.566$

0.424

$\mathrm{b}_{2}$

$\mathrm{P}_{\text {mild bleed }}$ (derived), d, $\%$

$-3.43$

39.6

$\mathrm{P}_{\text {moderate bleed }}$ (derived), de \%

55.7

$\mathrm{P}_{\text {severe bleed }}$ (derived), d, $\%$

4.72

Inter-individual variability

\begin{tabular}{lll}
\hline$\lambda, \% \mathrm{CV}$ & $136 \mathrm{~b}$ & $7.95^{\mathrm{c}}$ \\
$\mathrm{b}_{1}$ and $\mathrm{b}_{2}, \mathrm{SD}$ & $1.67^{\mathrm{b}}$ & $10.8^{\mathrm{c}}$ \\
\hline
\end{tabular}

$\mathrm{b}_{1}$ and $\mathrm{b}_{2}$ : baseline logits of the cumulative bleeding severity probabilities; PK: pharmacokinetics; $\mathrm{CL}$ : clearance; CV: coefficient of variation; IF50: factorVIII activity resulting in half-maximum inhibition; LBW: lean body weight; P: probability; Q: inter-compartmental clearance; RSE: relative standard error; RTTCE: repeated time-to-categorical event;SD: standard deviation;V1: central volume of distribution;V2: peripheral volume of distribution; $\lambda$ : bleeding hazard; $\gamma$ : shape factor of the Gompertz distribution.

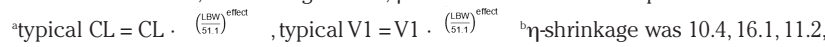
15.1 , and $26.7 \%$ on $\mathrm{CL}, \mathrm{V} 1, \mathrm{PK}$ residual error, $\lambda$ and b, respectively. ${ }^{\text {Relative standard }}$ error for inter-individual variability parameters reported on the approximate standard deviation scale (standard error/variance estimate)/2. 'Derivations and further details

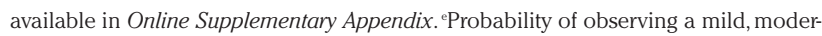
ate or severe bleed.

the covariates available and the model parameters. For PK, higher vWF levels were associated with a decreased FVIII CL; an expected finding since the complex FVIII-vWF is known to protect FVIII from proteolytic degradation. ${ }^{41}$ The impact was, however, modest, with the typical value of CL being 2.30 and $1.59 \mathrm{dL} / \mathrm{h}$ at vWF levels of $64 \%$ and $179 \%$ ( $5^{\text {th }}$ and $95^{\text {th }}$ percentiles). The magnitude of the vWF effect on CL is in agreement with results of other popula-

Table 3. Parameter estimates for the repeated time-to-categorical event (RTCE) sub-component of the final model, including only joint spontaneous bleeds.

Parameters Estimate RSE (\%)

RTTCE model (only joint spontaneous bleeds)

Structural model

$\lambda_{0.5 \mathrm{IU} / \mathrm{d},}$, year $^{-1}$

1.65

$\lambda_{20 \text { IU/dL, }}$ year ${ }^{-1}$

$\lambda_{\text {(0 IU/LL, 1 year) }}$ (derived) $)^{\mathrm{d}}$, year

IF50 (derived) $)^{\mathrm{a}}$, IU/dL

$\gamma$, year

$b_{1}$

$\mathrm{b}_{2}$

$\mathrm{P}_{\text {mild bleed }}$ (derived),, $\mathrm{ab} \%$

$\mathrm{P}_{\text {moderate bleed }}$ (derived),, $\mathrm{ab} \%$

$\mathrm{P}_{\text {severe bleed }}$ (derived), ${ }^{\mathrm{a}, \mathrm{b}} \%$

Inter-individual variability

\begin{tabular}{ccc}
$\lambda, \% \mathrm{CV}$ & $157^{\mathrm{c}}$ & $11.4^{\mathrm{d}}$ \\
$\mathrm{b}_{1}$ and $\mathrm{b}_{2}$, SD & $2.13^{\mathrm{c}}$ & $17.0^{\mathrm{d}}$ \\
\hline
\end{tabular}

$\mathrm{b}_{1}$ and $\mathrm{b}_{2}$ : baseline logits of the cumulative bleeding severity probabilities; $\mathrm{CV}$ : coefficient of variation; IF50: FVIII activity resulting in half-maximum inhibition; P: probability; RSE: relative standard error; RTTCE: repeated time-to-categorical event; SD: standard deviation; $\lambda$ : bleeding hazard; $\gamma$ : shape factor of the Gompertz distribution. The pharmacokinetics model was the same as presented in Table 2. ${ }^{a}$ Derivations and further details available in the Online Supplementary Appendix. ${ }^{\mathrm{b}}$ Probability of observing a mild, moderate or severe bleed. ' $\eta$-shrinkage was 21.8 , and $35.1 \%$ on $\lambda$ and $b$, respectively. ${ }^{~}$ Relative standard error for inter-individual variability parameters reported on the approximate standard deviation scale (standard error/variance estimate)/2.

tion PK studies. ${ }^{42,43}$ Interestingly, Black race was associated with a $27 \%(95 \% \mathrm{CI}: 12-40)$ lower CL compared to Caucasians. A similar trend was previously identified for a B-domain deleted recombinant FVIII product, ${ }^{6}$ and might be in part due to higher vWF levels in Black race patients or other unknown factors. ${ }^{44}$ Moreover, Asian race had a $14.2 \%$ (95\%CI: 2.0-28) higher CL compared to Caucasians. Such a trend had been identified during the previous development of the population PK model, but the relation was not retained due to lack to statistical significance. ${ }^{36}$ Due to the modest effect sizes, and the high uncertainty associated with the low number of patients of these races (Asian 31, Black 10), these correlations should be interpreted with caution.

The RTTCE component of the model describes the bleeding probability throughout the study period and the likelihood of that bleed to be mild, moderate or severe. The hazard function describes the distribution of bleeds in time, and represents the instantaneous risk of having a bleed per unit of time. Thus, the cumulative hazard gives the likely number of events in a given time interval. In this analysis, the hazard described a declining bleeding probability with time. The estimated lambda parameter $(\lambda)$ represents the bleeding hazard at the end of the LEOPOLD I and II studies (1 year), and the shape parameter $(\gamma)$ describes whether the hazard decreases, remains stable or increases with time. The bleeding hazard estimates for a typical patient at the end of the study were $2.7,2.0$ and 1.2 year- 1 for the thresholds of 1,5 and $15 \mathrm{IU} / \mathrm{mL}$, reflecting the model-predicted annual bleeding rates of patients constantly at a trough value. For instance, a typical patient with a permanent level of $1 \mathrm{IU} / \mathrm{dL}$ would be expected to have approximately three bleeds per year, if he remained 


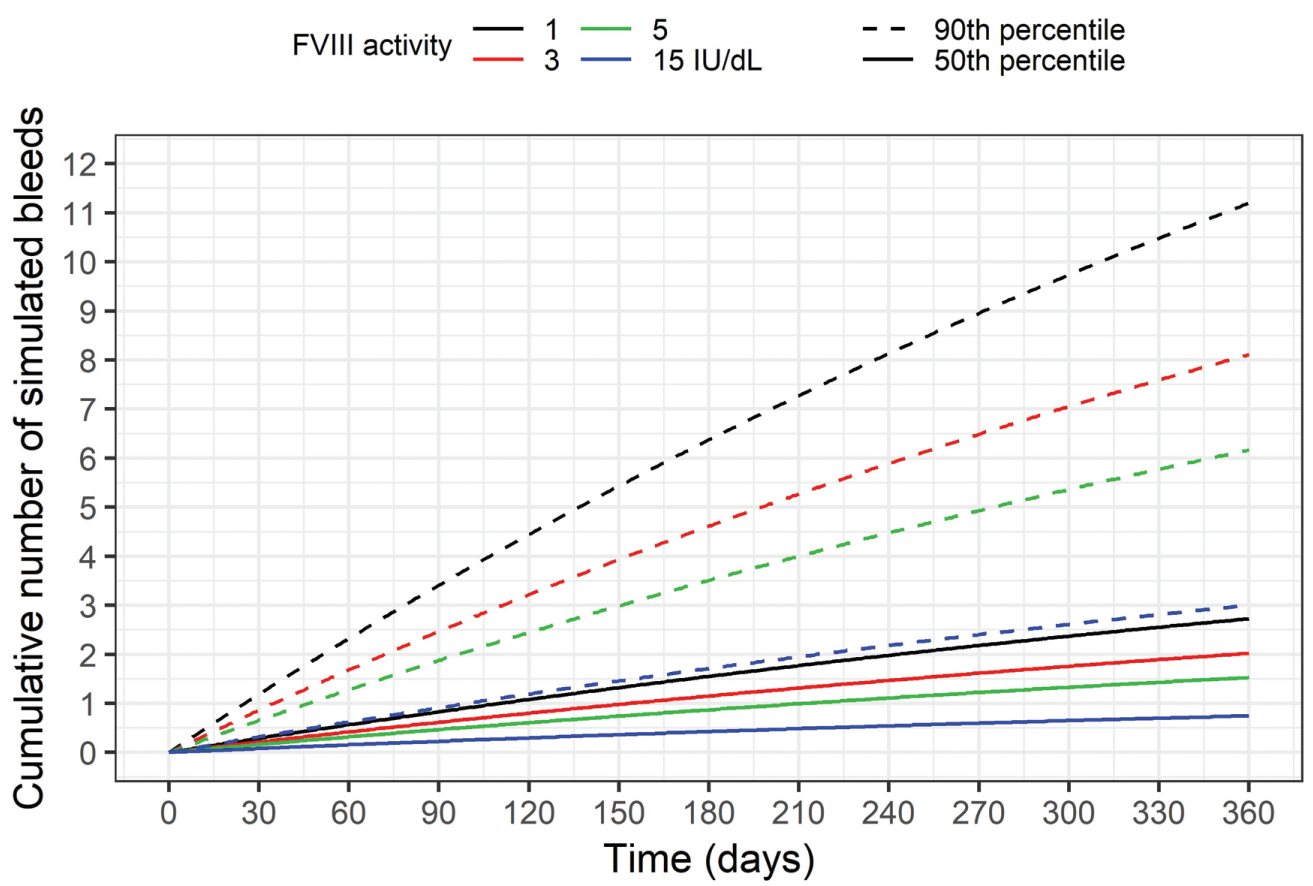

Figure 5 . The $50^{\text {th }}$ and $90^{\text {th }}$ percentiles of the cumulative number of simulated bleeds during 1 year after starting treatment assuming only inter-individual variability on the bleeding hazard unexplained by any covariates. The doses considered were 420 IU, $1260 \mathrm{IU}, 2100 \mathrm{IU}$ and $6240 \mathrm{IU}$ administered every two days, which correspond to trough factor VIII (FVIII) activity values at steady-state of 1, 3, 5 and $15 \mathrm{IU} / \mathrm{dL}$. Results based on 2000 simulations for a median patient weighing $60 \mathrm{~kg}$.

at the same hazard that was estimated at the end of the study. This numerical summary ignores protective effects against bleeds provided by higher levels and overall exposure, and therefore less bleeds would be expected in a realworld scenario. No additional correlations between PK and the bleeding hazard were found, besides the timevarying FVIII activity.

A time trend was identified in the hazard with a typical patient having 5.0 bleeds/year at the start of the study and 2.8 bleeds/year at the end (assuming a constant plasma FVIII activity of $0.5 \mathrm{IU} / \mathrm{dL}$ ). The lower bleeding rate at the end of the study most likely captures a treatment effect, not explained by plasma FVIII activity, and can be a consequence of a normalization of the clotting system due to prophylactic treatment or a better adherence to treatment in a clinical trial setting. The number of bleeds in the 12month pre-study period was found to be a strong predictor of the bleeding frequency. This finding can be explained by the individual-specific bleeding risk given, for example, by pre-existing joint disease and extent of joint damage, comorbidities or level of physical exercise.

The estimated unexplained inter-individual variability on the bleeding hazard was high (111\%), representing the variability in the bleeding tendency not being explained by the time-varying plasma FVIII activity or other covariates. Figure 5 shows that even when virtual patients with identical characteristics had a plasma FVIII activity trough of $5 \mathrm{IU} / \mathrm{dL}$, i.e. moderately above the common target of 1 IU/dL, the cumulative number of simulated bleeds was still highly variable at one year, with a median 1.53 bleeds, and $40 \%$ of the patients having $1.53-6.17$ bleeds, and $10 \%$ having more than 6.17 bleeds. Such high variability agrees with the clinical observation that patients may respond differently to identical plasma FVIII activity values, thus requiring also individual FVIII trough target lev- els. Part of this variability may be explained by factors that are known to influence the bleeding tendency in severe hemophilia A patients but that were not available to our study, such as FVIII gene mutation type, or physical activity patterns. ${ }^{45,46}$ However, even though the magnitude of inter-patient variability potentially explained by missing co-variates is unknown, variability in a real-world scenario is still expected to be high due, for example, to lack of adherence to treatment, or unidentified hemostatic factors playing a role in the occurrence of bleeds.

This study has limitations. First, the LEOPOLD trials were not designed for the aims of this post-hoc analysis or to characterize the individual bleeding pattern. Information, for example, on pre-existing joint disease, comorbidities or level of physical exercise before and during the trials could have been useful to better characterize the bleeding patterns. Second, bleeds (occurrence and severity) and doses were self-reported, which may result in uncertainty of timing and occurrence of bleeds (although the data were reviewed and validated by study staff in an effort to reduce uncertainty). Third, patients without any bleeds (33\% of those enrolled in the bleeding observation period) contributed with $\mathrm{PK}$ and co-variate information, but with little information to the characterization of the FVIII activity-bleeding hazard relationship. Finally, in the absence of data from on-demand treatment or placebo, we are not able to estimate a bleeding hazard corresponding to untreated patients. Thus, our results cannot be applied to treatment strategies other than prophylaxis.

The individual bleeding hazard estimated by the presented mathematical model is a numerical translation of the individual bleeding phenotype and may be used to further optimize the individualization of dosing regimens of replacement FVIII products. Using this model for dose 
individualization, a pre-defined FVIII activity target would not be required, in contrast to the current implementation of PK-based dosing. Instead, the full model would be employed using a Bayesian approach, with the estimation of the individual bleeding hazard in addition to the individual PK parameters. Thus, the individualized dose and dosing interval would be selected not only based on the individual pharmacokinetics, but also the individual bleeding risk. This implementation warrants further study; namely, to understand which information is required to allow a precise estimation of the individual bleeding risk and which target to aim for in the dose calculation. ${ }^{47}$

In conclusion, we have developed an integrated population PK and repeated time-to-categorical bleed model based on data from patients with severe hemophilia A fol- lowing prophylactic treatment. This combined model characterizes the relationship between the dose of a recombinant FVIII product, plasma FVIII activity, the bleeding outcome, as well as severity and the correlation with covariates. In the future, this model may be used for dose-tailoring using covariate, PK and/or bleeding information. Such an application requires a detailed theoretical assessment as well as robust data on bleeding and other factors, such as lifestyle or comorbidities, with the final goal of suggesting a more effective individualized FVIII dose.

\section{Acknowledgments}

The authors would like to thank Gunnar Yngman for skillful assistance during the implementation of the full random effects modeling approach.

\section{References}

1. Ahlberg A. Haemophilia in Sweden. VII. Incidence, treatment and prophylaxis of arthropathy and other musculo-skeletal manifestations of haemophilia A and B. Acta Orthop Scand Suppl. 1965:Suppl 77:73-132.

2. Geraghty S, Dunkley T, Harrington C, Lindvall K, Maahs J, Sek J. Practice patterns in haemophilia A therapy -- global progress towards optimal care. Haemophilia. 2006; 12(1):75-81.

3. Manco-Johnson MJ, Abshire TC, Shapiro $\mathrm{AD}$, et al. Prophylaxis versus episodic treatment to prevent joint disease in boys with severe hemophilia. N Engl J Med. 2007; 357(6):535-544.

4. Fischer K, Ljung R. Primary prophylaxis in haemophilia care: Guideline update 2016. Blood Cells Mol Dis. 2017;67:81-85.

5. Iorio A, Marchesini E, Marcucci M, Stobart $\mathrm{K}$, Chan AK. Clotting factor concentrates given to prevent bleeding and bleedingrelated complications in people with hemophilia A or B. Cochrane Database Syst Rev 2011;9:CD003429.

6. Abrantes JA, Nielsen EI, Korth-Bradley J, Harnisch L, Jonsson S. Elucidation of Factor VIII Activity Pharmacokinetics: A Pooled Population Analysis in Patients With Hemophilia A Treated With Moroctocog Alfa. Clin Pharmacol Ther. 2017; 102(6):977-988.

7. Collins PW, Fischer $K$, Morfini $M$, Blanchette VS, Bjorkman S, International Prophylaxis Study Group Pharmacokinetics Expert Working G. Implications of coagulation factor VIII and IX pharmacokinetics in the prophylactic treatment of haemophilia. Haemophilia. 2011;17(1):2-10.

8. Iorio A, Blanchette V, Blatny J, Collins P, Fischer K, Neufeld E. Estimating and interpreting the pharmacokinetic profiles of individual patients with hemophilia A or B using a population pharmacokinetic approach: communication from the SSC of the ISTH. J Thromb Haemost. 2017; 15(12):2461-2465.

9. Berntorp E. If you know you will also see: population pharmacokinetics is the way to personalize and optimize prophylaxis in hemophilia. J Thromb Haemost. 2017;15(6):1103-1105.

10. Stemberger M, Kallenbach F, Schmit E, et al. Impact of Adopting Population Pharmacokinetics for Tailoring Prophylaxis in Haemophilia A Patients: A Historically Controlled Observational Study. Thromb Haemost. 2018;119(3):368-376.

11. Sheiner LB, Beal S, Rosenberg B, Marathe VV. Forecasting individual pharmacokinetics. Clin Pharmacol Ther. 1979;26(3):294305.

12. Bjorkman S, Collins P. Measurement of factor VIII pharmacokinetics in routine clinical practice. J Thromb Haemost. 2013; 11(1):180-182.

13. Iorio A. Using pharmacokinetics to individualize hemophilia therapy. Hematology Am Soc Hematol Educ Program. 2017; 2017(1):595-604

14. Iorio A, Edginton AN, Blanchette V, et al. Performing and interpreting individual pharmacokinetic profiles in patients with Hemophilia A or B: Rationale and general considerations. Res Pract Thromb Haemost. 2018;2(3):535-548.

15. Collins PW, Blanchette VS, Fischer K, et al. Break-through bleeding in relation to predicted factor VIII levels in patients receiving prophylactic treatment for severe hemophilia A. J Thromb Haemost. 2009;7(3): 413-420.

16. Bjorkman S. Prophylactic dosing of factor VIII and factor IX from a clinical pharmacokinetic perspective. Haemophilia. 2003;9Suppl1:101-108; discussion109-110.

17. Valentino LA, Mamonov V, Hellmann A, et al. A randomized comparison of two prophylaxis regimens and a paired comparison of on-demand and prophylaxis treatments in hemophilia A management. J Thromb Haemost. 2012;10(3):359-367.

18. Ahnstrom J, Berntorp E, Lindvall K, Bjorkman S. A 6-year follow-up of dosing, coagulation factor levels and bleedings in relation to joint status in the prophylactic treatment of haemophilia. Haemophilia. 2004;10(6):689-697.

19. Aledort LM, Haschmeyer RH, Pettersson $\mathrm{H}$. A longitudinal study of orthopaedic outcomes for severe factor-VIII-deficient haemophiliacs. The Orthopaedic Outcome Study Group. J Intern Med. 1994;236(4): 391-399.

20. Petrini P. What factors should influence the dosage and interval of prophylactic treatment in patients with severe haemophilia A and B? Haemophilia. 2001;7(1):99-102.
21. Fischer K, Berntorp E. Targeting facto replacement therapy in severe hemophilia: which level is important? Semin Thromb Hemost. 2015;41(8):860-863.

22. Petrini P, Valentino LA, Gringeri A, Re WM, Ewenstein B. Individualizing prophylaxis in hemophilia: a review. Expert Rev Hematol. 2015;8(2):237-246.

23. Valentino LA, Pipe SW, Collins PW, et al. Association of peak factor VIII levels and area under the curve with bleeding in patients with haemophilia A on every third day pharmacokinetic-guided prophylaxis. Haemophilia. 2016;22(4):514-520.

24. Iorio $\mathrm{A}$, Iserman $\mathrm{E}$, Blanchette $\mathrm{V}$, et al. Target plasma factor levels for personalized treatment in haemophilia: a Delphi consensus statement. Haemophilia. 2017;23(3): e170-e179.

25. Chai-Adisaksopha C, Hillis C, Thabane L, Iorio A. A systematic review of definitions and reporting of bleeding outcome measures in haemophilia. Haemophilia. 2015;21(6):731-735.

26. Karlsson KE, Plan EL, Karlsson MO Performance of three estimation methods in repeated time-to-event modeling. AAPS J. 2011;13(1):83-91.

27. Cox EH, Veyrat-Follet C, Beal SL, Fuseau E, Kenkare S, Sheiner LB. A population pharmacokinetic-pharmacodynamic analysis of repeated measures time-to-event pharmacodynamic responses: the antiemetic effect of ondansetron. J Pharmacokinet Biopharm. 1999;27(6):625-644.

28. Juul RV, Rasmussen S, Kreilgaard M, Christrup LL, Simonsson US, Lund TM. Repeated Time-to-event Analysis of Consecutive Analgesic Events in Postoperative Pain. Anesthesiology. 2015;123(6):1411-1419.

29. Lindauer A, Laveille C, Stockis A. Time-toSeizure Modeling of Lacosamide Used in Monotherapy in Patients with Newly Diagnosed Epilepsy. Clin Pharmacokinet. 2017;56(11):1403-1413.

30. Plan EL, Karlsson KE, Karlsson MO Approaches to simultaneous analysis of frequency and severity of symptoms. Clin Pharmacol Ther. 2010;88(2):255-259.

31. Saxena K, Lalezari S, Oldenburg J, et al. Efficacy and safety of BAY 81-8973, a fulllength recombinant factor VIII: results from the LEOPOLD I trial. Haemophilia. 2016;22(5):706-712. 
32. Kavakli K, Yang R, Rusen $\mathrm{L}$, et al. Prophylaxis vs. on-demand treatment with BAY 81-8973, a full-length plasma proteinfree recombinant factor VIII product: results from a randomized trial (LEOPOLD II). J Thromb Haemost. 2015;13(3):360-369.

33. Ljung R, Kenet G, Mancuso ME, et al. BAY 81-8973 safety and efficacy for prophylaxis and treatment of bleeds in previously treated children with severe haemophilia A: results of the LEOPOLD Kids Trial. Haemophilia. 2016:22(3):354-360.

34. Shah A, Solms A, Garmann D, et al. Improved Pharmacokinetics with BAY 818973 Versus Antihemophilic Factor (Recombinant) Plasma/Albumin-Free Method: A Randomized Pharmacokinetic Study in Patients with Severe Hemophilia A. Clin Pharmacokinet. 2016;56(9):10451055.

35. Beal SL, Sheiner LB, Boeckmann AJ, Bauer RJ (eds). NONMEM 7.4 Users Guides. ICON plc, Gaithersburg, MD. (1989-2018). [https://nonmem.iconplc.com/nonmem743]

36. Garmann D, McLeay S, Shah A, Vis P, Maas Enriquez M, Ploeger BA. Population pharmacokinetic characterization of BAY 818973, a full-length recombinant factor VIII: lessons learned - importance of including samples with factor VIII levels below the quantitation limit. Haemophilia. 2017;23(4):528-537.

37. Agresti A. Modelling ordered categorical data: recent advances and future challenges. Stat Med. 1999;18(17-18):2191-2207.

38. Sheiner LB. A new approach to the analysis of analgesic drug trials, illustrated with bromfenac data. Clin Pharmacol Ther 1994;56(3):309-322.

39. Yngman G, Nyberg J, Jonsson N, Karlsson MO. Practical considerations for using the full random effects modeling (FREM) approach to covariate modeling. 2017. PAGE 26. Abstr 7365 [www.page-meeting.org/? abstract $=7365]$.

40. Karlsson MO. A full model approach based on the covariance matrix of parameters and covariates. 2012. PAGE 21. Abstr 2455 [www.page-meeting.org/?abstract=2455].

41. Weiss HJ, Sussman, II, Hoyer LW. Stabilization of factor VIII in plasma by the von Willebrand factor. Studies on posttransfusion and dissociated factor VIII and in patients with von Willebrand's disease. J Clin Invest. 1977;60(2):390-404.

42. Nestorov I, Neelakantan S, Ludden TM, Li $\mathrm{S}$, Jiang H, Rogge M. Population pharmacokinetics of recombinant factor VIII Fc fusion protein. Clin Pharmacol Drug Dev. 2015;4(3):163-174.

43. Zhang Y, Roberts J, Tortorici M, et al Population pharmacokinetics of recombinant coagulation factor VIII-SingleChain in patients with severe hemophilia A. J Thromb Haemost. 2017;15(6):1106-1114.

44. Conlan MG, Folsom AR, Finch A, et al Associations of factor VIII and von Willebrand factor with age, race, sex, and risk factors for atherosclerosis. The Atherosclerosis Risk in Communities (ARIC) Study. Thromb Haemost. 1993;70 (3):380-385.

45. Carcao MD, van den Berg HM, Ljung R, Mancuso ME. Correlation between phenotype and genotype in a large unselected cohort of children with severe hemophilia A. Blood. 2013;121(19):3946-3952.

46. Broderick CR, Herbert RD, Latimer J, et al. Association between physical activity and risk of bleeding in children with hemophilia. JAMA. 2012;308(14):1452-1459.

47. Abrantes JA, Solms A, Garmann D, Nielsen EI, Jönsson S, Karlsson MO. Bayesian forecasting utilizing bleeding information to support dose Individualization of factor VIII. CPT Pharmacometrics Syst Pharmacol. 2019;8(12):894-903. 